\title{
СОВРЕМЕННЫЕ ТЕЛЕКОММУНИКАЦИИ НА ОСТРИЕ ИНДУСТРИИ 4.0 - ВЛИЯНИЕ ИЗМЕНЕНИЯ СТРУКТУРЫ И ПОТРЕБЛЕНИЯ УСЛУГ НА ИТ-ПРОЕКТЫ И ЭКСПЛУАТАЦИЮ ЭКОСИСТЕМЫ КЛИЕНТА ОПЕРАТОРА СВЯЗИ
}

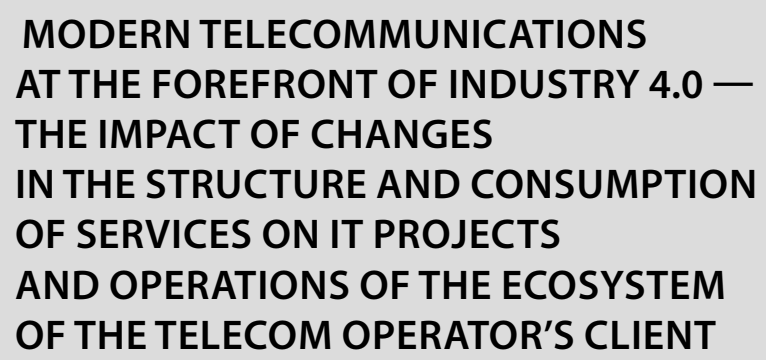

\section{S. Smirnov}

Summary. The article considers the problem of changing the demand and consumption structure of telecommunications services. The main factors that influence changes in the consumer's mindset and the structure of consumption of telecommunications services described in the literature are listed. Elements of the Industry 4.0 concept, their implementation by means of modern telecommunications, as well as additional factors that affect the infrastructure of the telecommunications environment from Industry 4.0 are considered. The main elements that make it possible to realize the customer's needs and customize (individualize) the product to meet their personal expectations are considered. The influence of these factors on the implementation of IT-projects on the implementation of changes or the introduction of new telecommunications and non-telecommunications products is considered. Using the system engineering method, key poorly formalized elements of project management and operation of the results of their implementation, which require special skills from the staff and special attention of the project Manager or product Manager, is identified. The task of developing multi-criteria analysis algorithms for automatic calculation of parameters of project management elements or functioning of components of the communication enterprise ecosystem that were previously calculated manually or evaluated based on expert opinions is formulated.

Keywords: telecommunications services, modern telecommunications, multi-criteria analysis, optimization algorithm, industry 4.0.

\author{
Смирнов Сергей Павлович \\ Аспирант, Российский технологический \\ университет МИРЭА (2. Москва) \\ spvert@mail.ru
}

Аннотация. В работе рассмотрена проблема изменения потребности и структуры потребления телекоммуникационных сервисов. Перечислены основные факторы, оказывающие влияние на изменении мировоззрения потребителя и структуры потребления телекоммуникационных услуг, описанные в литературе. Рассмотрены элементы концепции Индустрии 4.0, их реализация средствами современных телекоммуникаций, а также дополнительные факторы, оказывающие влияние на инфраструктуру телекоммуникационной среды со стороны Индустрии 4.0. Рассмотрены основные элементы, позволяющие реализовать потребности клиента, индивидуализировать продукт под его личные ожидания. Рассмотрено влияние перечисленных факторов на выполнение проектов, в области информационных технологий, по реализации изменений или внедрению новых телекоммуникационных и нетелекоммуникационных продуктов. С использованием метода системной инженерии выявлены ключевые плохо формализованные элементы управления проектами и эксплуатации результатов их выполнения, требующие специальных навыков от персонала и особого внимания менеджера проекта или менеджера продукта. Сформулирована задача разработки алгоритмов многокритериального анализа для автоматического расчета параметров элементов управления проектами или функционирования компонентов экосистемы предприятия связи, ранее рассчитывавшихся вручную или оценивавшихся на основе экспертных мнений.

Ключевые слова: телекоммуникационные услуги, современные телекоммуникации, многокритериальный анализ, алгоритм оптимизации, индустрия 4.0.

времени они выполняли роль обеспечения связи между конкретными людьми или группами людей для целей передачи коротких осмысленных сообщений. Взрывной рост телекоммуникаций относится к концу XX века и началу 2000-х годов: появление массового интернета и мобильной сотовой связи полностью преобразили облик 
современного мира. Современное состояние телекоммуникаций в России - это совершенно изменившийся мир и определяемое им новое мировоззрение. Повсеместное распространение сотовой связи ликвидировало подавляющее количество проблем, существовавших до середины 90-х годов. В настоящее время суммарный объем активных СИМ-карт российских сотовых операторов многократно превышает население России. Также, практически во всех городах практически все домовладения покрыты домашним интернетом по технологии FTTx. Широта покрытия и прошедшие ценовые войны привели к тому, что Интернет-канал, по сравнению с концом 20 века, перестал быть самостоятельной услугой и превратился, в определенном смысле, в такой же сервис как электричество, вода или канализация. В условиях, когда стоимость безлимитного мобильного интернета составляет, по состоянию на середину 2020 года, около 800 рублей, а безлимитный «домашний интернет» стоит от 400 рублей в месяц, борьба за клиента и его деньги ведется в направлении предоставления дополнительных услуг, полезного контента и удобства клиента. Клиент, в свою очередь тоже сильно изменился, стал существенно более требовательным и придирчиво выбирает товары или услуги, подходящие ему наилучшим образом [1][2].

Если применить системный подход [3-5] и оценить современные вызовы экосистемы современного человека к средствам производства, то можно сказать, что наблюдается [1]:

- Экспоненциальный рост сложности изделий даже самого простого бытового назначения [6]. Количество элементов в продукте массового использования, например, умном телевизоре или смартфоне, исчисляется десятками миллиардов [7];

- Высокая скорость изменения требований к целевому изделию [8][9], малый срок жизни требований [9][10], следование требований моде [11]. Это касается как пользовательских устройств, так и услуг связи и тарифов;

- Массовость производства изделий и вытекающие из этого массовость требований к изделию, что порождает большую вариативность требований [12];

- Преобразование финансовой модели - от владельца к инвестору, давление финансового рынка, сокращение срока жизни поколения изделия с десятилетий до полугода-года [13]. Существенное увеличение влияния цены изделия; повышенные требования к технологичности производства;

- Обучаемые системы и системы систем, принятие машиной не алгоритмизированных решений на основании анализа и статистики возмуще- ний-реакций, критическое влияние качества и объемов проводимого обучения [14][15][16];

- Этические нормы, поддержка выбора и принятия машиной решений в критических ситуациях, когда любой выбор приводит к нанесению того или иного объема ущерба [17][18]. Существенный рост влияния качества программного обеспечения;

- Технологический тупик, недостаточность неисследованных возможностей в существующих технологиях, потребность в новых технологиях [19][20];

- Экологичность. Ограниченность (конечность) ресурсов для производства, недостаточность и неэффективность мер по утилизации и обеспечению оборота редких и дорогостоящих ресурсов [21].

Для того, чтобы адресовать перечисленные факторы в контексте телекоммуникаций требуется принципиально новый подход, предлагаемый Индустрией 4.0 - не только в появление новых технологий, но и интеграция уже существующих в одну систему для достижения одной цели - улучшения качества жизни человека, создания условий, в которых человек может приобретать то что ему необходимо, а не то что ему предлагается.

Концепция Индустрии 4.0 включает в себя следующие ключевые компоненты, обеспечивающие формирование единого информационного пространства: управление жизненным циклом продукта (Product Lifecycle Management,PLM),BigData,продуманноепроизводство(S.M.A.R.T. factory), кибер-физические системы (Cyber-physical systems, CPS), Интернет вещей (IoT) и интероперабельность [22]. Современная телекоммуникационная индустрия обеспечивает техническую связность и среду передачи данных элементов в продуманном производстве, кибер-физических системах и интернете вещей, а также исходную информацию и инфраструктуру для BigData. В то же время, потребности в быстром создании новых уникальных продуктов, заставляют телекоммуникационную индустрию создавать внутри себя самостоятельные элементы PLM, BigData, S.M.A.R.T. Factory, ІоТ и обеспечивать интероперабельность сервисов. Таким образом, существенные изменения в требованиях к гибкости предлагаемых в телекоммуникационной сфере решений, фактически, требующие от оператора предоставлять настроенные под конкретного клиента услуги, более того, выявлять потребности клиента на основании его поведения или имеющейсяилиприобретаемойинформации-являются фактическими признаками, на основании которых телекоммуникационная сфера может рассматриваться как самостоятельный компонент Индустрии 4.0. Или можно сказать, что, де факто, телекоммуникационная 
индустрия, на данный момент, находится на лезвии Индустрии 4.0, являясь неотъемлемой ее частью [1].

Если рассмотреть визуализацию пирамиды потребностей Маслова в контексте цифрового мира, то мы легко можем увидеть, что, де факто, мы говорим о реализации потребностей человека с помощью некоторого набора инструментов: на базовом уровне потребностей мы предоставляем commodity сервисы [23] для обеспечения доступа в цифровой мир (средства доступа - электронные устройства, смартфоны, ноутбуки, планшеты; среды доступа сотовая связь, фиксированная связь, WiFi); на уровне базовых потребностей речь идет о предоставлении инструментов для он-лайн общения, некоторого базового контента, определенных профессиональных сервисов (например телемедицины или страховых продуктов для здоровья, для дома, для машины); на уровне психологических потребностей предоставляются инструменты в виде социальных сетей и сред общения и получения персональной обратной связи. Для каждой потребности есть свои инструменты с помощью которой эти потребности могут быть удовлетворены. И современный телеком должен отвечать на эти новые потребности и создавать уникальные персонализированные пользователем под себя продукты, фактически реализуя концепцию S.M.A.R.T factory, только не в мире производства собственной уникальной пары ботинок или костюма, а в цифровом мире. Как результат, в настоящий момент, в российской сфере телекоммуникаций очень активно развивается новый принцип взаимодействия с клиентов, который может быть описан как «Экосистема Клиента» - система отношений клиент-оператор, в рамках которой клиент может удовлетворить все или почтивсесвоицифровыепотребности. ТакаяЭкосистема включает в себя инструменты, агрегирующие различные элементы как телекоммуникационного плана, например, контент, streaming сервисы, площадки для общения, мессенджеры и т.п.; так и элементы из нетелекоммуникационного мира - аналитический контент, профессиональные сервисы (например, страхование, телемедицина, Uber для каких-то домашних работников), банковские и платежные сервисы, возможности универсальной идентификации и т.п. Как результат, клиент получает возможность собрать из предлагаемых компонентов пакет услуг, необходимый именно ему. И не только собрать, но и настроить под собственные нужды. Например, если клиент хочет смотреть только телеканал НВО и только на своем смартфоне, то он может исключить из своего пакета все остальные каналы и оптимизировать свои затраты как материальные так технические: нет нужно платить за контент, который клиент не потребляет, нет необходимости приобретать и развертывать элементы инфраструктуры (роутеры, например), которые не нужны. И в этом и состоит суть и главный результат Индустрии 4.0 для конечного пользователя: гибкое бережливое производство и доставка того, что требуется потребителю в те моменты, когда это требуется потребителю.

Необходимо отметить, что реализация инструментов и средств Индустрии 4.0 в телекоммуникационной области в силу сложности осуществляется с применением методов управления проектами. В контексте данной статьи говорится об ИТ-проектах: проектах по модернизации существующих или разработке новых компонент ИТ-архитектуры телекоммуникационного предприятия. Классическим подходом является выявление потребности бизнеса или клиента, описание этой потребности в формате формализованных требований, реализация изменений, интеграция (комплексирование), развертывание. Это может реализовываться как в классической водопадной модели и использованием, например, Rational Unified Process, так и с применением гибких подходов, с использованием фреймфорков SCRUM, Scaled Agile Framework (SAFe), Disciplined Agile Delivery (DAD), Dynamic System Development Method (DSDM), Feature Driven Development (FDD), Kanban, Lean SD, LeSS, или иных - в зависимости от объема и типа решаемой задачи. Но ключевым является то, что решается конкретная задача.

$\begin{array}{cccr}\text { B } & \text { рамках же концепции } & \text { Индустрии } \\ 4.0 & \text { телекоммуникационная отрасль } & \text { получает }\end{array}$ совершенно новый вызов - дать клиенту возможность получать необходимый ему продукт самостоятельно и научить его это делать - это современная потребность, которая требует серьезного переосмысления подходов, рекомендаций, лучших практик, формировавшихся в телекоммуникационной отрасли десятилетиями. Ключевыми элементами в реализации этой потребности становятся:

- Атомарные переиспользуемые системные компоненты (features), реализующие регулярную функциональность;

- Среднеуровневые компоненты, реализующие оркестрацию features для достижения целей бизнес-потребности;

- Высокоуровневые компоненты, создающие ценность для бизнеса (business capabilities);

- Инструменты и фреймворки, которые позволяют не терять широту обзора задачи при решении конкретной проблемы, а также управлять точностью и достоверностью исходных данных;

- Жесткие требования по обеспечению непрерывности бизнеса и непрерывности сервиса для клиента; 
- Таким образом, ключевыми факторами, специфичными для современного состояния телекоммуникационной индустрии, оказывающими существенное влияние на ИТ-проекты, являются:

- Требование гибкости продукта для клиента;

- Требование быстрого Time to Market (TTM) для продукта;

- Требование быстрого Time to Customer (TTC) для экземпляра продукта;

- Требование оптимальности затрат на создание продукта и вывод его на рынок (стоимости ТТМ);

- Требование оптимизации затрат на предоставление экземпляра продукта (стоимость ТТС);

- Требование непрерывности сервиса для клиента;

- Требование непрерывность бизнеса при внедрении нового продукта.

Ежегодно в мире инициируется и выполняется более миллионаИТ-проектовобщейстоимостьюболеетриллиона долларов США и лишь 30\% из них завершаются успешно, т.е. созданием продукта или услуги с ожидаемыми свойствами, в установленное время, в рамках установленного бюджета [24]. Анализ причин неуспешности проектов, например приведенный в современных обзорах [25-31], привел к появлению описанных методов и стандартов управления проектами, например PMBOK [32] или PRINCE2 [33], призванных увеличить процент успешности проектов. В то же время, процессы функционирования продуктов, их гибкости и удобства относятся не к сфере интересов проектного управления, а к сфере интересов системной инженерии, метод которой направлен на создание успешных систем, удовлетворяющих потребности заинтересованных сторон [3].

Исходя из этого, можно сделать вывод о необходимости разработки подходов и их реализации В инструментальных средствах для оптимизации ведения проектов по реализации компонент Индустрии 4.0 в сфере телекоммуникаций:

- Методы и механизмы для поддержки проектной деятельности по разработке и внедрению новых продуктов и элементов экосистемы потребителя (для элементов управления проектами и элементов системной инженерии);

- Методы и механизмы для оптимизации стоимостных характеристик эксплуатации продуктов и элементов экосистемы потребителя (для элементов системной инженерии).

Методы поддержки проектной деятельности описаны в виде стандартов или сводов знаний [32] [33] включают в себя рекомендации, фреймворки и процедуры, реализованные в многочисленных инструментах для поддержки управления проектами, например, Microsoft Project. Данные инструменты позволяют управлять расписанием, ресурсами проекта, фиксировать стоимостные параметры. В то же время, данные инструменты не позволяют решать плохо формализованные задачи, требующие существенного экспертного участия или особых навыков:

- Управление коммуникациями в части автоматизации обеспечения договоренности участников проекта и заинтересованных сторон на всем протяжении проекта;

- Управление рисками проекта в части автоматизации идентификации и оценки рисков проекта.

Методы оптимизации стоимостных характеристик эксплуатации продуктов и элементов экосистемы потребителя направлены на решение задачи поиска и выбора наиболее оптимального решения цепочки производства и поставок в рамках реализации S.M.A.R.T. factory при создании как продукта в целом так и его экземпляра, настроенного под конкретные потребности клиента.

Таким образом, разработка моделей и математических алгоритмов, позволяющих автоматизировать оптимизацию параметров создания и функционирования продукта для потребителя по множеству критериев, является актуальной задачей для исследований в настоящее время. Наиболее приоритетными представляются алгоритмы:

- Оптимизации управления коммуникациями в проекте и при эксплуатации продуктов в части возможности достижения консенсуса;

- Оптимизации управления рисками проектов по созданию новых продуктов или элементов экосистемы оператора связи в рамках Индустрии 4.0, а также при эксплуатации этих элементов и продуктов;

- Снижения стоимости проектов;

- Улучшения стоимостных характеристик при эксплуатации созданных элементов экосистемы оператора связи в телекоммуникационной сфере.

\section{Выво $\Delta \mathrm{b}$}

Таким образом, в работе

- Рассмотрена проблема изменения потребности и структуры потребления телекоммуникационных услуг.

- Перечислены основные факторы, оказывающие влияние на изменении мировоззрения потребителя и структуры потребления телекоммуникационных услуг, описанные в литературе.

- Рассмотрен состав концепции Индустрии 4.0, реализация элементов концепции средствами современных телекоммуникаций, а также дополнительные факторы, оказывающие влияние 
на инфраструктуру телекоммуникационной сферы со стороны Индустрии 4.0.

- Рассмотрено влияние факторов изменения потребностей и мировоззрения клиентов на выполнение ИТ-проектов по реализации изменений или внедрению новых телекоммуникационных и нетелекоммуникационных продуктов.

- Определено понятие «Экосистема Клиента» оператора связи.

- Выявлены ключевые плохо формализованные элементы управления проектами и эксплуатации результатов их выполнения, требующие специальных навыков от персонала и особого внимания менеджера проекта или менеджера продукта.

- Сформулирована задача разработки алгоритмов многокритериального анализа для автоматического расчета параметров выявленных элементов управления проектами или функционирования компонентов экосистемы предприятия связи, ранее рассчитывавшихся или оценивавшихся вручную.

\section{ЛИТЕРАТУРА}

1. Smirnov S.P., Telecom Stories from Russia: being on razor of Industry 4.0 // ADVISORS Webinar record [Электронный ресурc] // URL: https://www.youtube.com/ watch?v=0DZFsKC9vvo. (Дата обращения 18.10.2020).

2. Smirnov S.P., Telecom Stories from Russia: being on razor of Industry 4.0 // PMI Course // URL: https://ccrs.pmi.org/search/соurse/471606 (Дата обращения 14.10.2020).

3. Александр Косяков и др. Системная инженерия. Принципы и практика. М: ДМК Пресс, 2017.

4. О'Коннор Дж., Макдермотт И. Искусство системного мышления: необходимые знания о системах и творческом подходе к решению проблем. М.: Альпина Бизнес Букс, 2006.

5. Rl, Faulconbridge \& Ryan, Mike. (2014). Systems Engineering Practice.

6. Steinhart, Eric. (2020). Complexity. DOI: 10.1007/978-3-030-43052-8_2.

7. NVIDIA Drive Xavier SOC Detailed — A Marvel of Engineering, Biggest and Most Complex SOC Design To Date With 9 Billion Transistors. [Электронный ресурс]. // URL: https://wccftech.com/nvidia-drive-xavier-soc-detailed/ (Дата обращения 01.02.2020).

8. [Chemuturi, Murali. (2013). Requirements Change Management. DOI: 10.1007/978-1-4614-5377-2_8.

9. . Халл, К. Джексон, Д. Дик. Инженерия требований. М.ДМК Пресс, 2017.

10. Moorthy S. (2013). Lifecycle Challenges in Long Life and Regulated Industry Products. D0I: 10.1007/978-81-322-1050-4_66.

11. Sheridan Mandy \& Moore, Christopher \& Nobbs, Karinna. (2006). Fast fashion requires fast marketing: The role of category management in fast fashion positioning. Journal of Fashion Marketing and Management. 10.301-315. D01: 10.1108/13612020610679286.

12. Modrak Vladimir \& BEDNAR, Slavomir \& Marton David. (2015). Generating product variations in terms of mass customization. SAMI 2015 — IEEE13th International Symposium on Applied Machine Intelligence and Informatics, Proceedings. 187-192. D0I: 10.1109/SAMI.2015.7061873.

13. Tariq Amna \& Musleh, Noor \& Nobanee Haitham. (2020). Credit Unions' Business and Financial Models.

14. Бром А.Е., Белоносов К. Ю. Исследование проблем внедрения беспилотных автомобилей в экономическую среду // Вестник Московского государственного областного университета. Серия: Экономика. 2018. № 1. С. 23-32

15. Simic Danica \& Bacanin Nebojsa. (2019). The Ethics of Machine Learning. 478-484. D0I: 10.15308/Sinteza-2019-478-484.

16. Soh Julian \& Copeland, Marshall \& Puca, Anthony \& Harris Micheleen. (2020). Ethical Al, Azure Al, and Machine Learning. 10.1007/978-1-4842-5958-0_5.

17. Безопасность алгоритмов машинного обучения. Атаки с использованием Рython. [Электронный ресурc] // URL: https://habr.com/ru/company/dsec/ blog/437092/. (дата обращения 01.06.2020).

18. Этика роботов — можно ли убить одного, чтобы спасти пятерых? [Электронный ресурc].URL: https://habr.com/ru/post/76255/ (дата 0бращения 01.06.2020).

19. «Сколько стоит вертолет». [Электронный ресурс] URL: https://lenta.ru/articles/2017/01/18/helidev/ (дата обращения 10.03.2020).

20. «Что такое платформа автомобиля и как сегодня строят машины». [Электронный ресурc] URL: https://www.kolesa.ru/article/chto-takoe-platformaavtomobilja-i-kak-ih-segodnja-strojat-2014-11-21 (дата обращения 10.03.2020).

21. Грязный секрет Аpple, или как разработка редкоземельных элементов вредит экологии и подчиняет себе производство iPhone. [Электронный ресурс]. // URL: https://www.iphones.ru/iNotes/403115 (дата обращения 01.02.2020).

22. Шесть составляющих Индустрии 4.0 [Электронный ресурс]. // URL: http://www.plm.pw/2016/09/The-6-Factors-of-Industry-4.0.html (Дата обращения 01.11.2020).

23. Н. Карр. Блеск и нищета информационных технологий. М. Секрет фирмы, 2005.

24. The Standish Group report. Chaos. 2011. [Электронный ресурс] // URL: https://www.projectsmart.co.uk/white-papers/chaos-report.pdf. (Дата 0бращения 01.06.2020).

25. PMl's pulse of the profession: The high cost of low performance. 2014. [Электронный ресурс] // URL: https://www.pmi.org/-/media/pmi/documents/public/pdf/ learning/thought-leadership/pulse/pulse-of-the-profession-2017.pdf. (Дата обращения 01.06.2020).

26. PMl's pulse of the profession. 9th Global Project management survey. 2017. [Электронный ресурc] // URL: https://www.pmi.org/-/media/pmi/documents/public/ pdf/learning/thought-leadership/pulse/pulse-of-the-profession-2017.pdf. (Дата обращения 01.06.2020). 
27. PMI's pulse of the profession:Success in disruptive times. 2018. [Электронный ресурc] //URL: https://www.pmi.org/-/media/pmi/documents/public/pdf/learning/ thought-leadership/pulse/pulse-of-the-profession-2018.pdf. (Дата обращения 01.06.2020).

28. PMI' pulse of the profession: Requirements management — a core competency for Project and Program success. 2014 [Электронный pecypc] // URL: https://www. pmi.org/-/media/pmi/documents/public/pdf/learning/thought-leadership/pulse/requirements-management.pdf. (Дата обращения 01.06.2020).

29. Geneca (2011). Doomed From the Start? Why a Majority of Business and IT Teams Anticipate Their Software Development Projects Will Fail. // Geneca. Oakbrook terrace, IL.

30. The state of project management. Annual survey. 2018. [Электронный ресурc] // URL: https://www.wellingtone.co.uk/wp-content/uploads/2018/05/The-State-of-Project-Management-Survey-2018-FINAL.pdf. (Дата обращения 01.06.2020).

31. Gartner survey shows why project fails. 2012. [Электронный ресурс] // URL: https://thisiswhatgoodlookslike.com/2012/06/10/gartner-survey-shows-why-projects-fail/. (Дата обращения 01.06.2020).

32. A guide to the project management body of knowledge (PMBOK guide), 6th edition. 2017/ Newton-square, PA: Project management institute, 2017.

33. PRINCE2 [Электронный ресурc] // URL: https://www.prince2.com/eur/downloads. (Дата обращения 01.10.2020).

(c) Смирнов Сергей Павлович ( spvert@mail.ru).

Журнал «Современная наука: актуальные проблемы теории и практики»

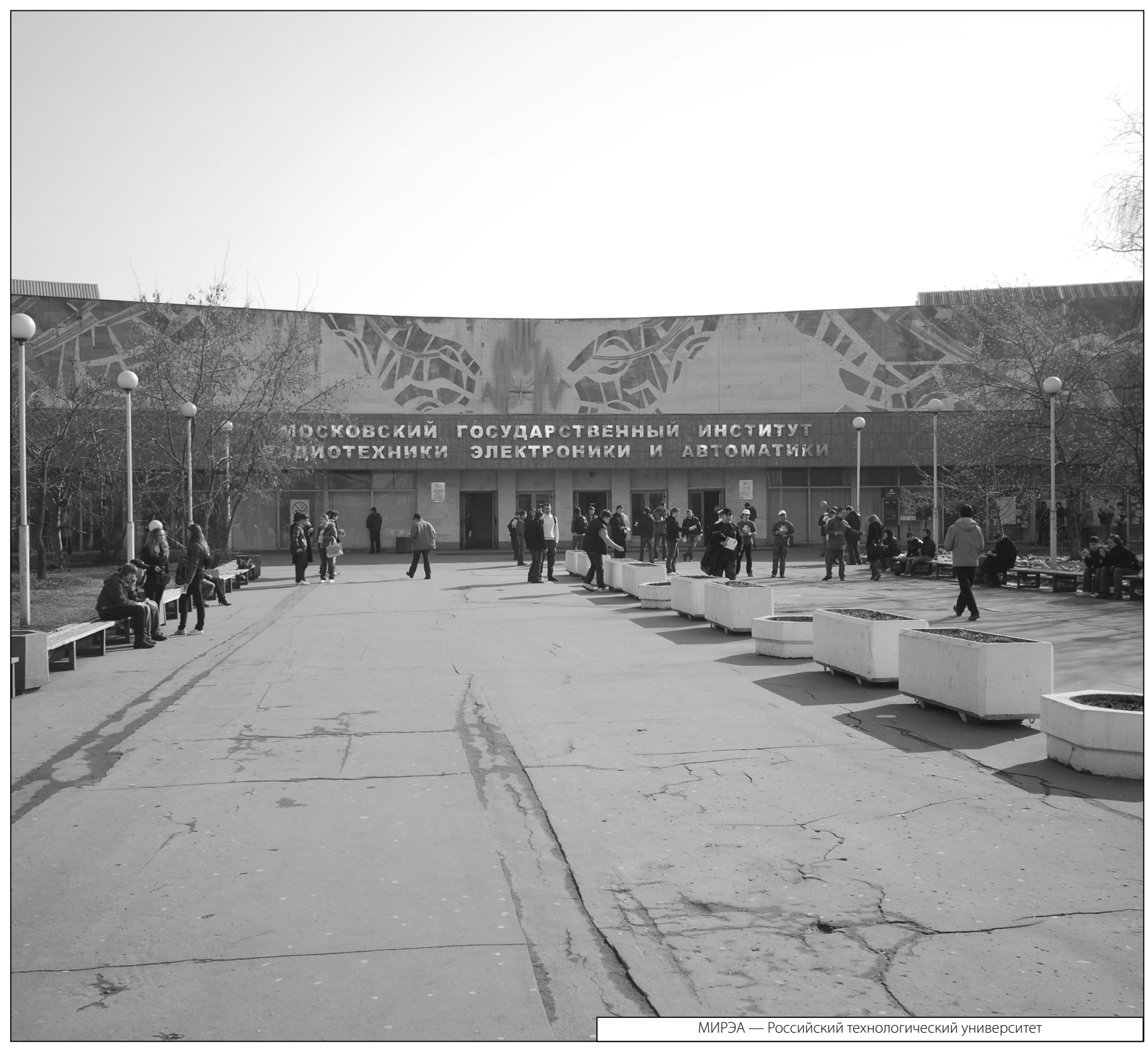

